EPJ Web of Conferences 66, 02059 (2014)

DOI: $10.1051 /$ epjconf/20146602059

(C) Owned by the authors, published by EDP Sciences, 2014

\title{
Coulomb excitation of exotic nuclei at REX-ISOLDE with MINI- BALL
}

\author{
Th. Kröll ${ }^{1, a}$ for the MINIBALL and REX-ISOLDE collaborations \\ ${ }^{1}$ Institut für Kernphysik, Technische Universität Darmstadt, Germany
}

\begin{abstract}
In this contribution nuclear structure studies with post-accelerated radioactive ion beams from the REX-ISOLDE facility at CERN are presented. The method employed is $\gamma$-ray spectroscopy with the MINIBALL array following "safe" Coulomb excitation. Recent results concerning the investigation of nuclear shapes are presented and discussed.
\end{abstract}

\section{Motivation}

Since Rutherford's epoch-making approach scattering experiments are a method of paramount importance for the investigation of nuclear properties. As targets of exotic short-lived isotopes cannot be made, the nuclei of interest have to be provided as radioactive ion beams (RIBs). The preparation of such beams at REX-ISOLDE (CERN) is discussed in Sect. 2.

In this contribution, we will focus on "safe" Coulumb excitation, the inelastic scattering due to a purely electromagnetic interaction. The cross section is mainly a measure for the electromagnetic transition probabilities. These are related to the "collectivity" of nuclear states, hence how many nucleons contribute to the excitation. The reproduction of shell closures, the "magic numbers", is a milestone for every nuclear theory. Small collectivities, corresponding to reduced transition strengths $B(E \lambda)$ of a few Weisskopf units, can be indications for shell closures and spherical shapes. Large $B(E \lambda)$ values accompanied by low excitation energies are typical for regions of the nuclear chart without shell closures and nuclei with deformed shapes. Therefore, the experimental results challenge the predictions from nuclear theory for the appearance and disappearance of shell closures going away from the valley of $\beta$-stability.

\section{REX-ISOLDE facility}

ISOLDE at CERN is the world-leading ISOL ("Isotope Separation OnLine") facility with more than 45 years of experience in producing low-energy radioactive ion beams (RIBs) [1]. So far, more than 1000 different isotopes have been delivered to experiments. The $1.4 \mathrm{GeV}$ proton beam from the PS Booster impinges on a thick production target, for most cases discussed in this contribution consisting of $\mathrm{UC}_{x} /$ graphite $\left(\approx 50 \mathrm{~g} / \mathrm{cm}^{2}{ }^{238} \mathrm{U}\right)$. Alternatively, the protons irradiate a Ta or $\mathrm{W}$ rod next to the production target and the generated neutrons induce the fission of the uranium. Compared to the direct proton impact, the composition of the cocktail of fragments is changed. The produced nuclei

\footnotetext{
ae-mail: tkroell@ikp.tu-darmstadt.de
} 
diffuse within the heated target and reach the ion source. Here, a selection of the chemical element is intended. Sometimes it is possible to ionise only a certain isotope or even a single long-lived state of the nucleus. The highest selectivity is offered by the RILIS ("Resonant Ionisation Laser Ion Source"). The singly charged ions are extracted and sent through one of the two mass separators available at ISOLDE (GPS and HRS). In principle, the selection of the chemical element and the mass allows for isotopically pure beams. However, most of the beams contain a cocktail of different isobars because non-selective thermal ionisation is unavoidable. After passing the separator, a 30-60 keV low-energy beam can be sent to the experiments.

The idea of the REX-ISOLDE facility has been to post-accelerate these beams enabling nuclear reaction studies [2]. Firstly, the ions are cooled and bunched in a penning trap (REXTRAP). Then, they are sent to a second trap where the ions are charge bred, i.e. their charge state is increased by collisions with an intense electron beam (REX-EBIS). At a mass to charge ratio of about $A / q=4$, the ions are injected into a linac (REX-LINAC) where the energy is boosted to currently up to $3 \mathrm{MeV} / \mathrm{u}$. REX-ISOLDE has been operated successfully for more than 10 years and post-accelerated ISOL beams of nearly 100 isotopes of elements ranging from Li to Ra have been delivered to experiments.

\section{Experimental setup}

The most important instrument for the study of exotic nuclei at REX-ISOLDE is the highly efficient MINIBALL spectrometer consisting of 8 triple clusters of six-fold segmented HPGe detectors. MINIBALL was the first large $\gamma$-ray spectrometer equipped with fully digital electronics. The data acquisition system is operated in a timestamp mode. The $\gamma$-rays are usually measured in coincidence with beam- and target-like nuclei detected by arrays of segmented Si detectors. This could be a CD-shaped DSSSD (double-sided segmented Si strip detector) or the T-REX array [3].

However, as isobaric contaminants in the beam excite the target as well the beam composition has to be determined. Different methods have been developed for this task comprising switching on/off the RILIS, stopping the beam inside MINIBALL and analysing the characteristic decay $\gamma$-rays, or using a $\Delta E-E$ telescope or a Bragg detector mounted in the beam dump downstream of MINIBALL.

A comprehensive description of the experimental setup can be found in Ref. [4].

\section{Safe Coulomb excitation}

Many experiments at REX-ISOLDE with MINIBALL employ $\gamma$-ray spectroscopy following "safe" Coulomb excitation as a tool to study collective properties of exotic nuclei. Here, the beam energy is chosen such that projectile and target interact only via the well-known electromagnetic interaction. The radioactive isotope of interest impinges as beam on a target and either of them (or both) can be excited. From the double-differential cross sections, extracted from the measured $\gamma$-ray-particle yields, the electromagnetic matrix elements are determined. As the cross section depends on both transitional but also diagonal matrix elements (deorientation), reduced transition probabilities $B(E \lambda)$ as well as spectroscopic electric moments $Q_{\lambda}$ can be extracted. In the analysis, the experimental data are compared with results of calculations solving a system of coupled differential equations to obtain the cross sections for a set of matrix elements.

The sensitivity of the cross section on the different matrix elements changes with the scattering angle. Therefore, the data set set is divided in subsets each covering a smaller range of scattering angles. Each subset is treated as independent measurement. The matrix elements are varied until a consistent reproduction of the observed data is obtained applying the maximum likelihood method, see e.g. [5]. Additional information, like lifetimes from independent experiments can be considered too. 
Figure 1 illustrates the method for a simple generic case, the excitation of the first $2^{+}$state in an even-even nucleus where only one transitional $M_{02}=\left\langle 2^{+}|M(E 2)| 0^{+}\right\rangle$and one diagonal $M_{22}=$ $\left\langle 2^{+}|M(E 2)| 2^{+}\right\rangle \propto Q_{2}\left(2^{+}\right)$matrix element are considered. Multiple excitation to higher-lying states, e.g. the first $4^{+}$state, are neglected. The values of the matrix elements and their errors are obtained from the projections of the $1 \sigma$ contour onto the axes.
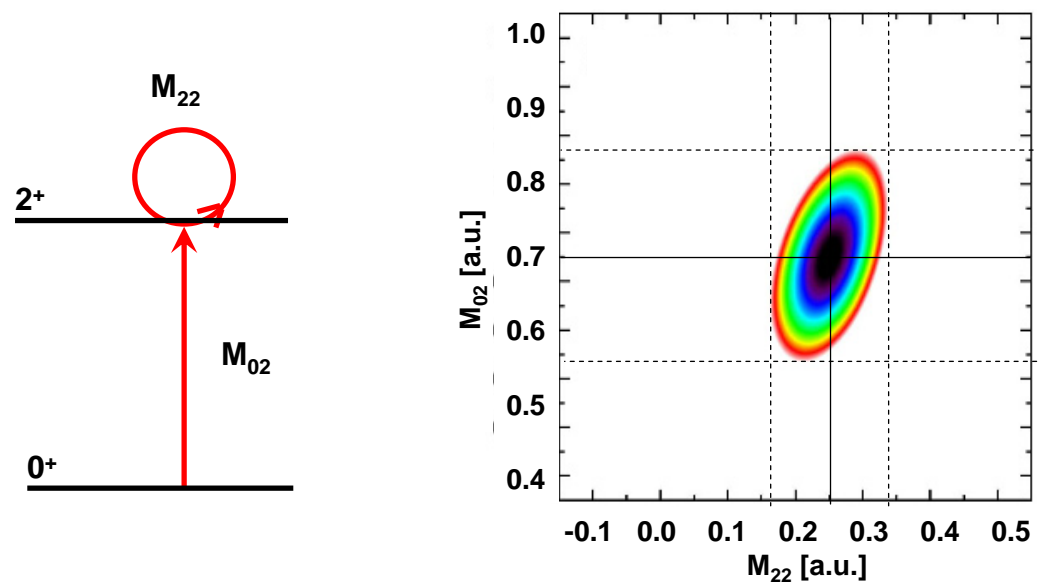

Figure 1. Coulomb excitation of a $2^{+}$state involving a transitional $M_{02}$ and a diagonal $M_{22}$ matrix element (left). The values and their errors are determined by the maximum likelihood method, see text, projecting the $1 \sigma$ contour onto the axes (right).

The use of a target nucleus with known properties allows for a analysis of the projectile excitation relative to the target excitation avoiding the necessity to determine absolute efficiencies and beam intensities. However, the beam composition is needed. Other observables, like lifetimes or $g$ factors, have been measured too by adapting methods used with stable beams to the requirements for experiments at REX-ISOLDE.

\section{Recent results on nuclear shapes}

The shape of a nucleus is one of its basic features. Doubly-magic nuclei exhibit spherical shapes, whereas most other nuclei are more or less deformed. The quadrupole and higher order terms in the residual interaction are responsible for this effect. Hence, the study of nuclear shapes allows for conclusions on the underlying microscopic structure and the relevant interactions.

The shape of nuclei evolves with proton or neutron number along isotopic, isobaric or isotonic chains. The nuclei may even undergo a shape phase transition. In some nuclei also states of different shape occur at similar excitation energies, the so-called phenomenon of shape coexistence. This illustrates the importance of studies to determine the shape of nuclei as fingerprint of nuclear structure effects.

For stable or isomeric states, electric moments can be extracted also from high-resolution laser spectroscopy of hyperfine states, whereas for short-lived states the deorientation effect in Coulomb excitation is the method of choice to obtain these quantities. In the following, we will present some recent examples studied at REX-ISOLDE with MINIBALL. 


\subsection{Onset of deformation in $\mathrm{Kr}$ at $\mathrm{N}=60$}

The $A \approx 100$ mass region around ${ }^{100} \mathrm{Zr}$ is one of the regions of the nuclear chart where the phenomenon of a shape phase transition occurs [6]. Along the isotopic chains of $\operatorname{Zr}(Z=40)$ and $\operatorname{Sr}(Z=38)$ a sudden transition from almost spherical to strongly deformed shapes happens between $N=58$ and $N=60$. For $\operatorname{Kr}(Z=36)$ the candidate for the $2^{+}$state at a low excitation energy of $241 \mathrm{keV}$ seemed to indicate a similar behaviour.

At REX-ISOLDE the isotopes ${ }^{94,96} \mathrm{Kr}$ have been studied in projectile Coulomb excitation [7]. It was found that the excitation energy $E\left(2^{+}\right)=554 \mathrm{keV}$ in ${ }^{96} \mathrm{Kr}$ is only slightly lower than in ${ }^{94} \mathrm{Kr}$ $(666 \mathrm{keV})$. The $B\left(E 2,2^{+} \rightarrow 0^{+}\right)$increases from $19.5 \mathrm{~W} . u$. to 33.4 W.u., hence much slower compared to $\mathrm{Zr}$ and $\mathrm{Sr}$ where a jump by a factor of about 4 is observed. Hence, no sudden onset of deformation occurs in $\mathrm{Kr}$ at $N=60$ which is consistent with recent mass and charge radii measurements. This behaviour is also in agreement with calculations within the IBA-2 with a proton-neutron interaction based on the (constrained) Hartree-Fock-Bogoliubov approach using the microscopic Gogny-D1M energy density functional [7].

Interestingly, the nuclei change shape from a oblate deformation in ${ }^{94} \operatorname{Kr}\left(Q_{2}=-0.26_{-0.16}^{+0.19} e \mathrm{~b}\right)$ to a slightly prolate shape in ${ }^{96} \mathrm{Kr}\left(Q_{2}=0.15(53) \mathrm{eb}\right)$. Note, that the large error for ${ }^{96} \mathrm{Kr}$ is consistent also with spherical or oblate shapes. This agrees well with the interpretation of the charge radii of the ground states indicating a stabilising effect of oblate deformations along the $\mathrm{Kr}$ chain.

\subsection{Quadrupole collectivity around ${ }^{132} \mathrm{Sn}$}

The isotope ${ }^{132} \mathrm{Sn}$ is the heaviest doubly-magic nucleus below ${ }^{208} \mathrm{~Pb}$ which is experimentally accessible. As the astrophysical $r$ process is expected to pass via this region, the understanding of the nuclear structure is also important for modelling the nucleosynthesis. For Sn and Te, an asymmetric behaviour of excitation energies and quadrupole collectivity, represented by $B\left(E 2,0^{+} \rightarrow 2^{+}\right)$values, with respect to $N=82$ has been oberved. At REX-ISOLDE, $\mathrm{Cd}, \mathrm{Xe}$ and $\mathrm{Ba}$ isotopes have been investigated to extend such studies.

Just two protons below the shell closure at $Z=50$, the $\mathrm{Cd}$ isotopes are of particular interest. The even isotopes ${ }^{122-128} \mathrm{Cd}$ and the odd ${ }^{123} \mathrm{Cd}$ have been investigated at REX-ISOLDE [8-13]. It has been evidenced that the quadrupole collectivity of ${ }^{122,124} \mathrm{Cd}$ is enhanced to values above the predictions by state-of-the-art shell model calculations whereas for ${ }^{126} \mathrm{Cd}$ good agreement is found. The $B\left(E 2,0^{+} \rightarrow 2^{+}\right)$value in this isotope is confirmed by an independent lifetime measurement applying DSAM ("Doppler shift attennuation method") performed with MINIBALL at REX-ISOLDE [12]. For the isotopes ${ }^{122,124,126} \mathrm{Cd}$, the quadrupole moments of the first excited $2^{+}$states are very small, the nuclei are rather spherical [11]. A special case is the isotope ${ }^{128} \mathrm{Cd}$ whose $E\left(2^{+}\right)$anomaly can be interpreted so far only by beyond-mean-field calculations which predict a prolate deformation for this nucleus. Our study of ${ }^{128} \mathrm{Cd}$ will be discussed in detail in the contribution by S. Bönig to this conference [10]. Recently, the studies have been extended to odd isotopes. This will help to attribute the enhanced quadrupole collectivity below $A=126$ to certain single-particle orbitals [13].

As for the Xe isotopes $[8,9]$, a regular behaviour with respect to $N=82$ is observed for the $E\left(2^{+}\right)$energies and the $B(E 2)$ values in the Ba isotopes [14]. Here, the first combined analysis of the cross sections in Coulomb excitation and a lifetime measurement applying DSAM both performed

with MINIBALL at REX-ISOLDE has been done. However, the quadrupole moments in ${ }^{140,142} \mathrm{Ba}$ are considerably larger compared to the nearly spherical shapes just below $N=82$ [14]. 


\subsection{Shape coexistence in neutron-deficient $\mathrm{Hg}$, Po and $\mathrm{Rn}$ isotopes}

A fascinating phenomenon is the shape coexistence in the region of neutron-deficient $\mathrm{Hg}$ to $\mathrm{Rn}$ isotopes, see e.g. [15]. Most prominent example is the isotope ${ }^{186} \mathrm{~Pb}$ with two excited deformed $0^{+}$ states, one prolate and one oblate, which coexist with the spherical ground state within only $800 \mathrm{keV}$ energy difference, hence less than $1 \%$ of the total binding energy. The microscopic nature of this phenomenon is still not completely understood yet.

At REX-ISOLDE, beams of $\mathrm{Hg}$, Po and $\mathrm{Rn}$ became recently available allowing to perform Coulomb excitation experiments. So far, ISOLDE is the only facility worldwide which can deliver such heavy radioactive beams beams at low energies. The even-even isotopes ${ }^{182,184,186,188} \mathrm{Hg}$, 196,198,200,202,206 Po and ${ }^{202,204,206,208,210} \mathrm{Rn}$ have been investigated [16, 17].

Most advanced is the analysis of the $\mathrm{Hg}$ isotopes [16]. In $\mathrm{Hg}$, the level schemes are characterised by a ground state band whose level energies stay almost constant all along the isotopic chain (except at $N=126$ ). A second rotational band based on an intruder configuration is well separated from the ground state band by more than $1 \mathrm{MeV}$ around $A \approx 190$. This drastically changes going towards neutron deficient isotopes. At $A \approx 184$ the difference is only around $200 \mathrm{keV}$. In ${ }^{182} \mathrm{Hg}$, the second $0^{+}$ state is even below the first $2^{+}$state. In these nuclei, of course, a mixture between the two bands can be expected.

In our analysis, in ${ }^{182,184} \mathrm{Hg}$ a weakly deformed oblate shape in the ground state band has been observed as predicted assuming a $2 \mathrm{~h}$ configuration, hence two proton holes in the $Z=82$ shell. The first excited band, whose microscopic structure is expected to have a $4 \mathrm{p}-6 \mathrm{~h}$ configuration with four protons excited across the $Z=82$ shell gap, exhibits a much larger deformation. So far, no states corresponding to a $2 \mathrm{p}-4 \mathrm{~h}$ configuration have been observed. Theory predicts for the $4 \mathrm{p}-6 \mathrm{~h}$ configuration a prolate shape, however the experimental data do not exclude triaxiality, a further degree of freedom describing nuclear shapes. A more detailed analysis of the data is ongoing.

\subsection{Octupole states in ${ }^{222} \mathbf{R n}$ and ${ }^{224} \mathbf{R a}$}

Besides quadrupole deformation, octupole deformation resulting in reflection-asymmetric "pear shapes" is a further degree of freedom of the nuclear many-body system.

Strong octupole collectivty may occur if near to the Fermi surface orbitals which differ in angular momentum by $\Delta L=3$ and in spin by $\Delta S=0$ come next to each other. In order to highlight the collective character of the first $3^{-}$state, the squared matrix elements shown in Figure 2 (values taken from Ref. [18]) are divided by the excitation energy. Clearly, three regions of the nuclear chart can be identified around $Z \approx 34, N \approx 56, Z \approx 56, N \approx 88$ and $Z \approx 88, N \approx 134$, the "magic" octupole numbers, with the largest value known so far for ${ }^{226} \mathrm{Ra}$.

In order to investigate their octupole collectivity, $\mathrm{Rn}$ and $\mathrm{Ra}$ isotopes have been studied in Coulomb excitation. The even isotopes ${ }^{220} \mathrm{Ra}$ and ${ }^{224} \mathrm{Ra}$ were the first isotopes for which a $B(E 3)$ value has been measured at REX-ISOLDE [19]. From our results, we can conclude that ${ }^{224} \mathrm{Ra}$ has a larger octupole collectivity than ${ }^{220} \mathrm{Rn}$. The octupole moment of ${ }^{220} \mathrm{Rn}$, extracted from the transition strengths, $Q_{3}=2180 \mathrm{efm}^{3}$ is comparable to values in ${ }^{208} \mathrm{~Pb},{ }^{230,232} \mathrm{Th}$ or ${ }^{234} \mathrm{U}$ known to be rather octupole vibrational than statically octupole deformed. ${ }^{224} \mathrm{Ra}$ has a larger value of $Q_{3}=2980 \mathrm{efm}^{3}$, only slightly smaller than that of ${ }^{226} \mathrm{Ra}$, which can be interpreted as an indication for a rigid octupole deformed shape [19].

Odd nuclei with octupole deformation are of great interest for the search for atomic electric dipole moments (EDM) as the effect will be largely enhanced. Atomic EMDs are important to test CPT symmetry in nature as their existence would violate either $\mathrm{T}$ or $\mathrm{CP}$ symmetry and would constrain physics beyond the standard model [20]. At REX-ISOLDE, we have recently studied as first odd 


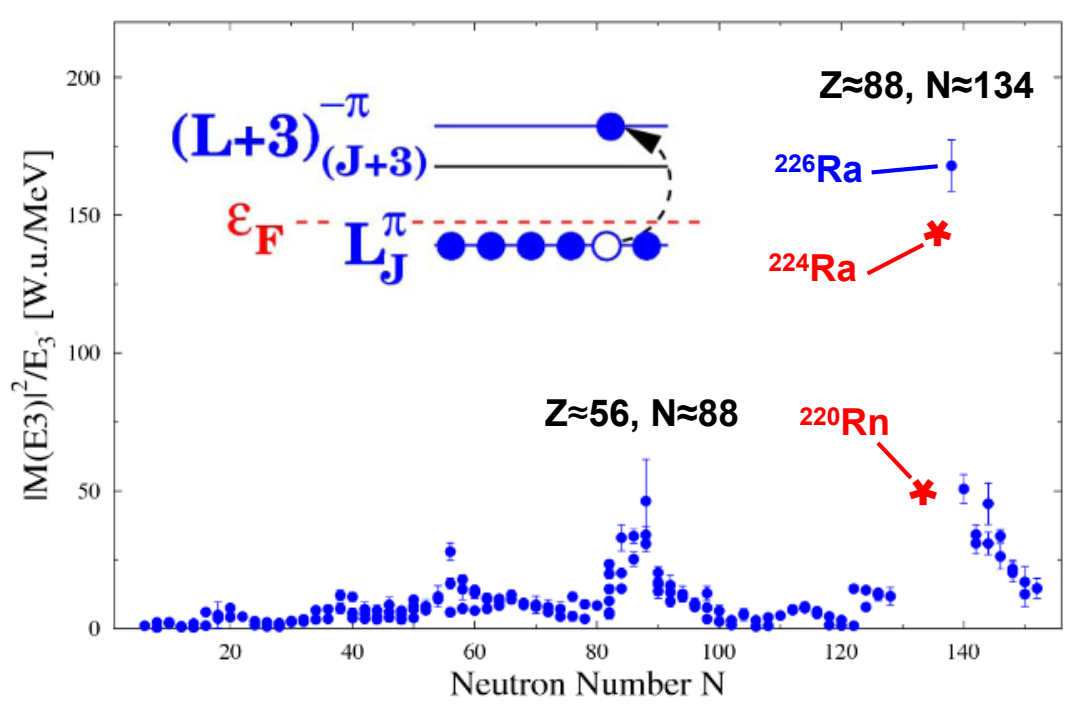

Figure 2. Square of octupole matrix elements divided by the excitation energy of the first $3^{-}$state. Our new values for ${ }^{220} \mathrm{Rn}$ and ${ }^{224} \mathrm{Ra}$ are included (plot by courtesy of M. Scheck).

nucleus the isotope ${ }^{221} \mathrm{Rn}$ in Coulomb excitation [21]. The results will help to guide future EDM searches. However, from the properties of the even isotopes it seems that e.g. ${ }^{225} \mathrm{Ra}$ may be a more promising candidate than ${ }^{221} \mathrm{Rn}$.

\section{Summary and Outlook}

The concept of the REX-ISOLDE facility at CERN, the post-acceleration of low-energy ion beams from ISOLDE, has been proven to be highly successful. With a broad range of isotopes at energies of up to $3 \mathrm{MeV} / \mathrm{u}$ available, many aspects and questions concerning the structure of exotic nuclei far-off the valley of stability have been addressed. Most important method is the $\gamma$-ray spectroscopy following "safe" Coulomb excitation and, more recently, nucleon-transfer reactions. The experimental setup consisting of the efficient high-resolution HPGe spectrometer MINIBALL in combination with arrays of segmented Si detectors is a powerful and versatile instrumentation to perform such studies.

In the near future REX-ISOLDE will be upgraded step-wise to HIE-ISOLDE [22], see also contribution by M. Borge to this conference [23]. From 2015, beam energies of up to $5.5 \mathrm{MeV} / \mathrm{u}$ will be available for experiments. In a second step, the energy will be increased further up to $10 \mathrm{MeV} / \mathrm{u}$. This is achieved by replacing the resonators of the REX linac with superconducting cavities. Additionally, higher beam intensities, e.g. because of an upgrade of the PS-Booster, and an improved beam quality are envisaged.

The higher beam energy will allow to extend the physics programme considerably. In Coulomb excitation experiments with heavy targets, the probability for multi-step processes as well as for onestep excitation of high-lying states will be strongly enhanced.

Besides Coloumb excitation, transfer reactions will profit as at HIE-ISOLDE such reactions can be studied also with heavy beams, some of them are unique to ISOLDE as low-energy beams. Such 
experiments aim at the investigation of single-particle properties in heavy nuclei and the results are often complementary to those obtained with Coulomb excitation.

These developments will extend the physics programme conducted at ISOLDE to new horizons and the study of exotic nuclei at CERN has a bright future.

\section{Acknowledgements}

This contribution represents the work of many groups, in particular students and postdocs, from all over Europe. I would like to mention M. Albers (Universität zu Köln), T. Behrens (TU München), C. Bauer, S. Bönig, A.-L. Hartig, C. Henrich, S. Ilieva, M. Scheck, R. Stegmann, M. Thürauf (TU Darmstadt), N. Bree, K. Wrzosek-Lipska (KU Leuven), T. Grahn (University of Jyväskylä), L. P. Gaffney and G. O'Neill (University of Liverpool) whose work is described in this proceeding.

The success of REX-ISOLDE is owed to the large commitment of the ISOLDE and REX teams resulting in a continuous improvement of the facility. This is accompanied by a constantly upgraded setup consisting of MINIBALL, the Si detectors and further auxiliary devices as well as the development of improved data analysis techniques.

This work has been supported by BMBF (No. 06 DA 9036I and No. 05 P12 RDCIA), HIC for FAIR, EU through EURONS (No. 506065) and ENSAR (No. 262010) and the MINIBALL and REX-ISOLDE collaborations.

\section{References}

[1] http://isolde.web.cern.ch

[2] D. Habs et al., Nucl. Instr. and Meth. B 139, 128 (1998)

[3] V. Bildstein et al., Eur. Phys. J. A 48, 85 (2012)

[4] N. Warr et al., Eur. Phys. J. A 49, 40 (2013)

[5] Review of Particle Physics, Phys. Lett. B 667, 13 (2008)

[6] H. Mei et al., Phys. Rev. C 85, 034321 (2012)

[7] M. Albers et al., Phys. Rev. Lett. 108, 062701 (2012); Erratum: Phys. Rev. Lett. 109, 209904 (2012); Nucl. Phys. A 899, 1 (2013)

[8] Th. Kröll et al., Eur. Phys. J. Special Topics 150, 127 (2007)

[9] T. Behrens, PhD thesis (TU München, 2009)

[10] S. Bönig et al., contribution to this conference

[11] S. Ilieva et al., to be published

[12] M. Thürauf, MSc Thesis (TU Darmstadt, 2012)

[13] A.-L. Hartig et al., ISOLDE Newsletter Spring 2013

[14] C. Bauer et al., Phys. Rev. C 86, 034310 (2012); PhD Thesis (TU Darmstadt, 2013)

[15] R. Julin et al., J. Phys. G 27, R 109 (2001)

[16] N. Bree, K. Wrzosek-Lipska et al., to be published

[17] T. Grahn et al., to be published

[18] T. Kibédy, R. H. Spear, Atomic Data and Nuclear Data Tables 80, 35 (2002)

[19] L. P. Gaffney et al., Nature 497, 199 (2013)

[20] T. Chupp et al., contribution to this conference

[21] G. O’Neill et al., ISOLDE Newsletter Spring 2013

[22] http://hie-isolde.web.cern.ch/hie-isolde/

[23] M. Borge et al., contribution to this conference 
